\title{
193-nm Photodissociation of Ions from Saturated and Unsaturated Aliphatic Molecules
}

\author{
Douglas J. Beussman, Tina A. Erickson and Christie G. Enke \\ Department of Chemistry, University of New Mexico, Albuquerque, New Mexico, USA
}

\begin{abstract}
To determine the analytical utility of photodissociation as a general fragmentation technique for tandem mass spectrometry of organic ions, the ability to fragment those ions considered least likely to absorb photons efficiently was investigated. To this end, the ability to photodissociate ions of aliphatic compounds by using 193-nm photons has been studied. Three fragment ions, the $\mathrm{C}_{4} \mathrm{H}_{9}^{+}$ion from $n$-hexane, the $\mathrm{C}_{4} \mathrm{H}_{7}^{+}$ion from 2-hexene, and $\mathrm{C}_{4} \mathrm{H}_{5}^{+}$ from 2-hexyne, have been photodissociated. The fragmentation efficiencies for all three ions studied were between 25 and $45 \%$. The photofragment ion spectrum for each precursor ion studied is made up of characteristic fragments. These spectra demonstrate the ability to photodissociate aliphatic ions that originate from both saturated and unsaturated molecules. This provides substantial hope that virtually all organic ions will be able to be photodissociated by using 193-nm photons. (J Am Soc Mass Spectrom 1996, 7, 114-117)
\end{abstract}

$\mathrm{R}$ ecently we reported the construction of a tandem time-of-flight (TOF) mass spectrometer designed to obtain tandem mass spectrometry spectra from compounds that elute from a gas chromatograph [1]. This instrument uses photo-induced dissociation (PID), or photodissociation, to impart energy into the precursor ions to cause fragmentation. This requires that the ions of interest absorb the photons at the laser wavelength used. For PID to be useful as a general fragmentation technique, all ions of interest must be able to be dissociated and, thus, there must exist a wavelength at which all ions of interest fragment.

Much is known about molecular absorption at wavelengths greater than $200 \mathrm{~nm}$, and molecular chromophoric groups with characteristic absorptions have been well studied [2-5]. Ion chromophoric groups that absorb below $200 \mathrm{~nm}$ have not been studied and little is known about the absorption of ions at these wavelengths. Other researchers have used 193-nm photons for photodissociation of organic ions, but all the ions studied have had groups recognized as chromophoric in their respective molecules. Bowers et al. [6] performed photodissociation of the bromobenzene molecular ion by using 193-nm photons. Peptide ions, which have numerous chromophoric groups such as amide linkages and aromatic side chains, have been photodissociated by using 193-nm light by numerous groups, which include Hunt et al. [7], Lebrilla et al. [8], Williams et al. [9], Martin et al. [10], and Gorman and Amster [11]. Recently Gimon-Kinsel et al. [12] reported

Address reprint requests to Dr. C. G. Enke, Department of Chemistry, University of New Mexico, 103 Clark Hall, Albuquerque, NM 87131. the photodissociation of peptides in a time-of-flight mass spectrometer. Although no product ions were collected, photodissociation was inferred by the observation of a reduction in precursor ion signal when the laser was discharged. We previously showed photodissociation results by using 193-nm photons for ions with various molecular chromophores, such as aromatic, amide, and alkene functionalities [13], as well as for peptides [14].

To investigate the possibility of the use of $193-\mathrm{nm}$ photodissociation as a generally applicable fragmentation technique for tandem mass spectrometry, we wanted to determine if even-electron ions that originate from saturated alkane molecules could be photodissociated. Because these ions (such as $\mathrm{C}_{4} \mathrm{H}_{9}^{+}$) have no functional group commonly thought of as a chromophore and because the photodissociation process requires that a photon be absorbed by the ion to impart energy and cause fragmentation, the applicability of photodissociation to aliphatic ions from saturated alkane molecules was not known.

\section{Experimental}

The tandem time-of-flight instrument used for these studies has been described previously [1]. Ions are formed by using an electron ionization (EI) source and are focused temporally at the ion-photon interaction region. The precursor ion packet of interest is selected by using an ion-deflection gate [15] prior to the interaction region. This allows other mass-to-charge ratio ion packets from the normal EI spectrum to be deflected and thus eliminates convolution with the product ion spectrum. The laser discharge is timed so that the 
photons and precursor ion packet reach the interaction region at the same time, which allows for energy deposition and ion fragmentation. The product ions and unfragmented precursor ions are then accelerated to impart mass-dependent velocities and are focused temporally at the detector position by a novel, broad energy range focusing reflectron [16].

The laser used is an excimer laser (Lambda-Physik, Acton, MA) that employs the 193-nm (ArF) line, with a pulse width of approximately $15 \mathrm{~ns}$. At this wavelength, the laser provides pulse energies between 50 and $275 \mathrm{~mJ}$. The beam is focused by a cylindrical plano-convex lens to a $1-\mathrm{mm}$-wide $\times 2-\mathrm{cm}$-high area at the interaction region. The various laser pulse energies used for the power studies are obtained by variation of the high voltage applied to the thyratron via the laser control program. The absorbance by the air, focusing lens, and laser windows has been accounted for by determination of the actual pulse energies delivered to the interaction region for the various laser output pulse energies. The laser manufacturer specifications for pulse-to-pulse energy stability is $\pm 5 \%$ for the wavelength used.

\section{Results and Discussion}

To determine the effects that varying degrees of unsaturation have on the photodissociation process, we studied the photodissociation of ions that result from the introduction of $n$-hexane, 2-hexene, and 2-hexyne into the EI source. Because the base peak in the normal EI spectrum of hexane is $m / z 57$, we decided to use the $m / z 57,55$, and 53 series from $n$-hexane, 2-hexene, and 2-hexyne, respectively. These mass-to-charge ratio values represent ions from a saturated molecule $\left(\mathrm{C}_{4} \mathrm{H}_{9}^{+}\right)$, a molecule with one unsaturation $\left(\mathrm{C}_{4} \mathrm{H}_{7}^{+}\right)$, and a molecule with two unsaturations $\left(\mathrm{C}_{4} \mathrm{H}_{5}^{+}\right)$. Because this instrument was designed to obtain tandem mass spectrometry data, the precursor ions studied were even-electron fragment ions from the normal EI mass spectrum instead of the odd-electron molecular ions.

The photofragment ion spectra (product spectra) for the photodissociation of $m / z 57$ from $n$-hexane, $m / z$ 55 from 2-hexene, and $m / z 53$ from 2-hexyne are shown in Figure 1. All of these spectra were collected by using laser pulse energies of $175 \mathrm{~mJ}(86 \mathrm{~mJ}$ at the interaction region, which corresponds to an energy density of $430 \mathrm{~mJ} / \mathrm{cm}^{2}$ ). To improve signal-to-noise ratios, each of these spectra is the average of 500 individual spectra. Because the ion packets are constantly traversing the flight path, each ion packet interacts with a single 15-ns laser discharge (unlike experiments in ion trap instruments where the ions are confined to a small region and multiple laser pulse interactions can occur with the same ion set to increase the fragmentation efficiency). Thus, averaging spectra from multiple laser discharges does not enhance the
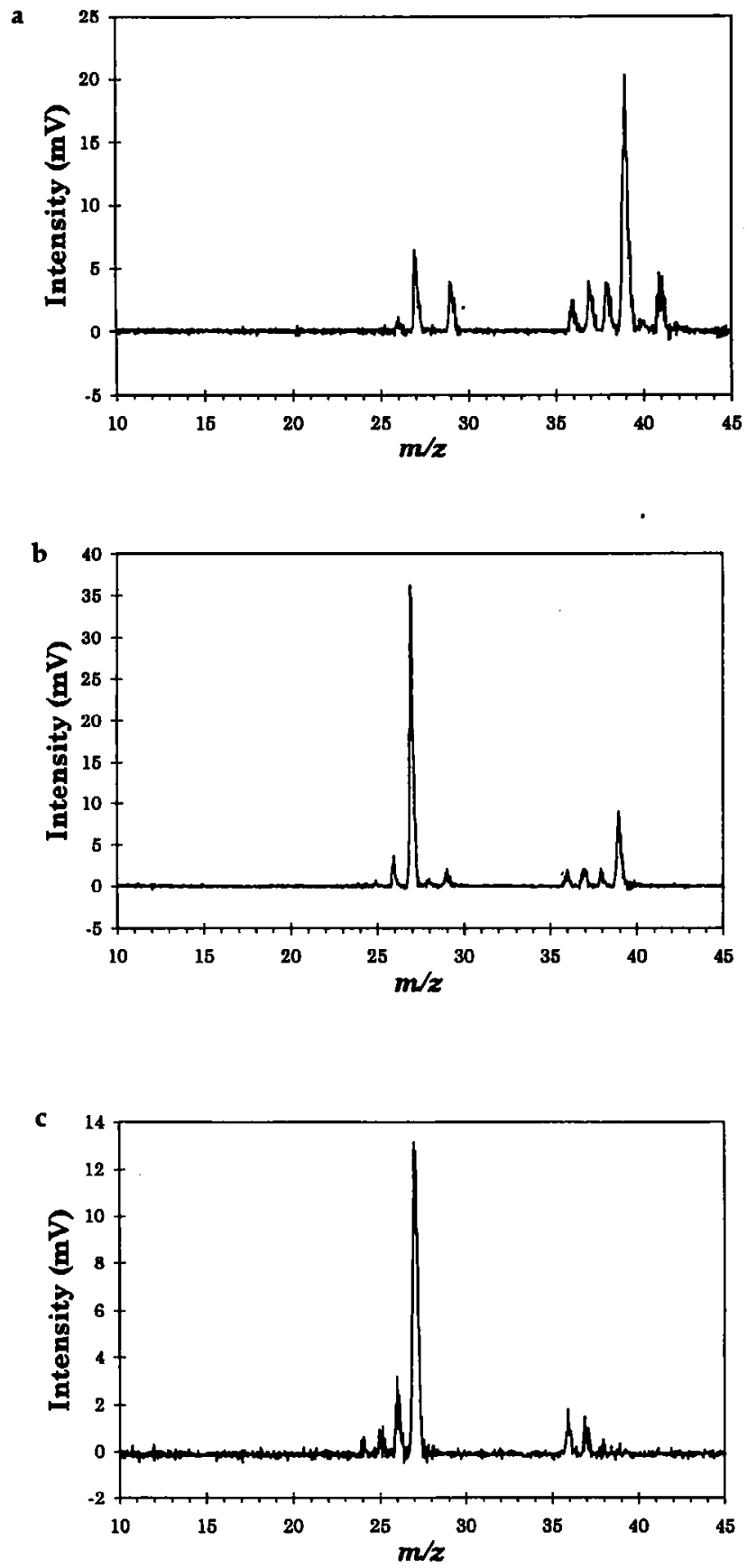

Figure 1. Photofragment ion spectra from PID by using 193-nm photons (175 mJ per laser pulse) of aliphatic ions (a) from $m / z 57$ from hexane, (b) from $m / z 55$ from 2-hexene, and (c) from $m / z$ 53 from 2-hexyne. Five hundred transients were summed for each spectrum.

fragmentation efficiency; it only serves to enhance the signal-to-noise ratio. As can be seen, all three ions were dissociated by using 193-nm photons, which yield characteristic product ions. The major ions in the $n$ hexane $m / z 57$ photofragment ion spectrum are at $m / z 27,39$, and 41. Fragmentation of $m / z 55$ from 2-hexene yields primarily $m / z 27$ and 39 product ions with no $m / z 41$, whereas the photofragment ion spectrum from $m / z 53$ of 2-hexyne is primarily $m / z 27$ with no $m / z 39$ or 41 . 
The spectra in Figure 1 indicate that photodissociation by using 193-nm photons may not require a chromophoric functionality, or conversely, that $\mathrm{C}-\mathrm{C}$ and/or $\mathrm{C}-\mathrm{H}$ bonds of alkane ions act as chromophores for 193-nm photons. This indicates that 193$\mathrm{nm}$ photodissociation may be useful as a general fragmentation technique for organic ions, because virtually all organic ions have these sorts of alkane-like bonds. Johnston and co-workers $[7,8]$ used 193-nm photons to photodissociate neutral molecules, which include alkanethiols [7] and alkenes [8]. These molecules have functional groups that may absorb the photons, but based on their results and on our observed fragmentation of ions of aliphatic molecules, we expect that virtually all polyatomic organic ions will absorb 193-nm light and fragment.

The effect of laser pulse energy on the photodissociation of the $\mathrm{m} / \mathrm{z} 53$ precursor ion can be seen in Figure 2. The percent precursor ion intensity depleted versus laser pulse energy (corrected for absorption by optics) is a linear function as shown in Figure 2a. The percent depletion versus laser pulse energy plots for the other two ions studied are similar to that shown in Figure 2a. All three graphs show a linear relationship between percent depletion of the precursor ion intensity and laser pulse energy, which indicates that all three fragmentations are single photon processes. Figure $2 b$ is a log- $\log$ plot of the data from Figure $2 a$ with a slope of 1.03, which also indicates a single photon event. Thus, because one photon corresponds to $6.4 \mathrm{eV}$, the photodissociation of the precursor ions requires no more than $6.4 \mathrm{eV}$. The power studies also indicate that significant fragmentation occurs with laser pulse energies as low as $50 \mathrm{~mJ}(33 \mathrm{~mJ}$ at the interaction region, which corresponds to an energy density of $165 \mathrm{~mJ} / \mathrm{cm}^{2}$ ), as can be seen in Figure 2a.

The bond strengths for normal, single bonds in organic molecules are almost all less than the $6.4 \mathrm{eV}$ $(147.6 \mathrm{kcal} / \mathrm{mol})$ that the photon deposits into the ion [19]. Based on this, it is not unexpected that the absorption of a single photon is sufficient to cause ion dissociation at a number of bond sites. Ab initio calculations are being performed in this laboratory to determine the energy required to produce the ion fragmentations observed.

The fragmentation efficiencies for the three ions studied at $175 \mathrm{~mJ}(86 \mathrm{~mJ}$ at the interaction region, which corresponds to an energy density of $430 \mathrm{~mJ} / \mathrm{cm}^{2}$ ) are presented in Table 1, as well as the photoinduced dissociation cross sections. These PID cross sections $(\sigma)$ are calculated by

$$
\sigma=\frac{\% \text { precursor ion depletion }}{\phi}
$$

where $\phi$ is the photon density at the interaction region.

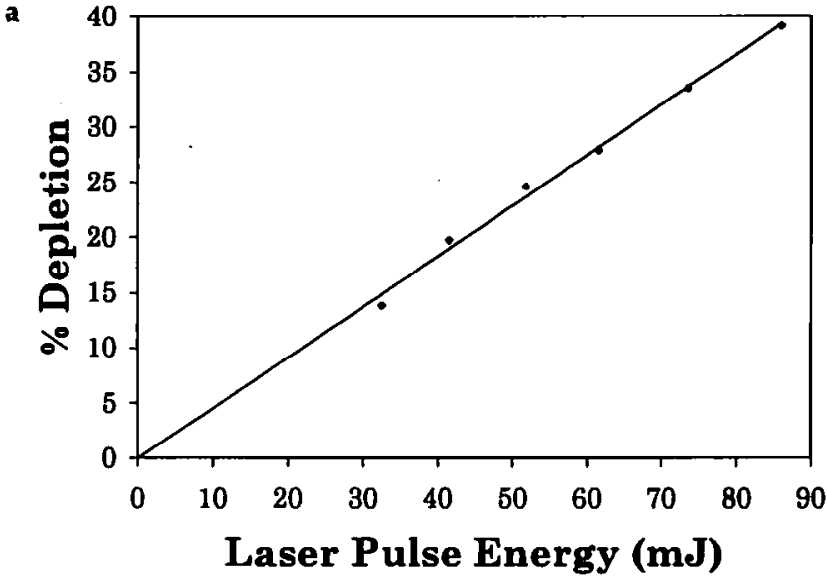

- Data Points - Linear Regression Fit

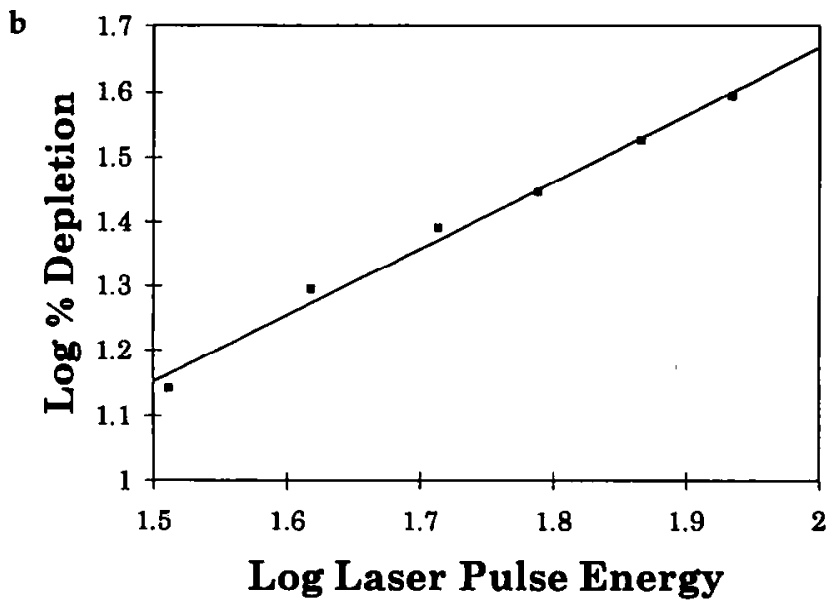

Figure 2. (a) Percent depletion of $m / z 53$ from 2-hexyne precursor ion intensity versus laser pulse energy (corrected for optic absorbances). (b) $\log -\log$ plot of data from Figure 2a.

There are at least four processes that normally occur to enable an ion to be dissociated by using PID: absorption of a photon, conversion of the photon energy to vibrational energy, distribution of the vibrational energy, and fragmentation of a bond that has excess vibrational energy. Direct absorption to a dissociative state is possible, as is bond dissociation prior to energy distribution, but these processes are rare. An enhance-

Table 1. Percent depletion of precursor ion intensity by using a 175-mJ laser pulse and calculated PID cross section for aliphatic ions studied by photodissociation by using 193-nm photons

\begin{tabular}{ccccc}
\hline Molecule & lon $m / z$ & $\begin{array}{c}\text { Ion } \\
\text { formula }\end{array}$ & $\begin{array}{c}\text { \% Depletion } \\
\text { (at } 175 \mathrm{~mJ})\end{array}$ & $\begin{array}{c}\text { PID cross section } \\
\left(\mathrm{cm}^{2}\right)\end{array}$ \\
\hline \hline n-Hexane & 57 & $\mathrm{C}_{4} \mathrm{H}_{9}^{+}$ & 20.5 & $7.4 \mathrm{E}-19$ \\
2-Hexene & 55 & $\mathrm{C}_{4} \mathrm{H}_{7}^{+}$ & 22.1 & $7.9 \mathrm{E}-19$ \\
2-Hexyne & 53 & $\mathrm{C}_{4} \mathrm{H}_{5}^{+}$ & 38.2 & $1.4 \mathrm{E}-18$ \\
\hline
\end{tabular}


ment of the first, second, or fourth process would lead to a higher fragmentation efficiency (and thus a higher PID cross section), as would a decrease in the third process. Thus, any of the preceding four processes, singly or in combination, could be responsible for the fact that the PID cross sections slightly increase with increasing number of ion unsaturations. Also, the energy imparted to the ion must increase the ion internal energy above the dissociation limit and the dissociation kinetics must allow the dissociation to occur within the $0.7 \mu \mathrm{s}$ of ion flight time between excitation and postdissociation acceleration. Theoretical considerations of the nature of the absorption process are underway in this laboratory.

A comparison of the initial peak area of the precursor ion with the sum of the peak areas for the undissociated precursor ion and the product ions indicates that all of the fragments formed in the PID process are detected. As can be seen, fragmentation efficiencies of approximately $20-40 \%$ for every laser pulse-source extraction event are observed with laser pulse energies of $175 \mathrm{~mJ}$ (energy density of $430 \mathrm{~mJ} / \mathrm{cm}^{2}$ in the interaction region). These efficiencies are comparable to those obtained by collision-induced dissociation (CID) in a triple quadrupole mass spectrometer [20].

\section{Conclusions}

We have demonstrated that ions from both saturated and unsaturated aliphatic molecules can be fragmented by using photodissociation with 193-nm photons. This observation raises the expectation that PID at $193 \mathrm{~nm}$ will prove to be a generally applicable method for tandem mass spectrometry of organic ions without the need for chromophoric derivatization. All three ions studied have comparable PID cross sections, which indicates a similarity in the photodissociation process for these ions at $193 \mathrm{~nm}$. The high PID fragmentation efficiencies for all three ions studied are comparable to those achieved in current commercial tandem mass spectrometry instruments employing CID.

Photodissociation is a highly advantageous ion fragmentation technique for tandem mass spectrometry instruments based on time-of-flight mass analyzers. The energy and time of the fragmentation event are well controlled and the high photon-ion overlap results in a high efficiency process. The ability to fragment a selected ion packet efficiently and to efficiently detect the resulting product ions as well as the demonstrated general applicability of the PID process should encourage further development of this approach.

\section{Acknowledgment}

We gratefully acknowledge the National Institutes of Health for supporting this work (NIH GM 44077).

\section{References}

1. Beussman, D. J.; Vlasak, P. R.; McLane, R. D.; Seeterlin, M. A.; Enke, C. G. Anal. Chem. 1995, 67, 3952-3957.

2. Dunbar, R. C. In Gas Phase Ion Chemistry, Vol. 2; Bowers, M. T., Ed.; Academic Press: New York, 1979; pp 181-220.

3. Harris, F. M.; Beynon, J. H. In Gas Phase Ion Chemistry, Vol. 3; Bowers, M. T., Ed.; Academic Press: New York, 1984; pp 99-128.

4. Levsen, K. In Advances in Mass Spectrometry 1985; Todd, J. F., Ed.; Wiley: New York, 1986; pp 359-377.

5. van der Hart, W. J. Int. I. Mass Spectrom. Ion Processes 1992, 118/119, 617-633.

6. Bowers, W. D.; Delbert, S.-S.; Mclver, R. T., Jr. Anal. Chem. 1986, 58, 969-972.

7. Hunt, D. F.; Shabanowitz, J.; Yates, J. R., III J. Chem. Soc., Chem. Commun. 1987, 548-550.

8. Lebrilla, C. B.; Wang, D. T.-S.; Mizoguchi, T. J.; McIver, R. T., Jr. J. Am. Chem. Soc. 1989, 111, 8593.

9. Williams, E. R.; Furlong, J. J. P.; McLafferty, F. W. J. Am. Soc. Mass Spectrom. 1990, 1, 288-294.

10. Martin, S. A.; Hill, J. A.; Kittrell, C.; Biemann, K. J. Am. Soc. Mass Spectrom. 1990, 1, 107-109.

11. Gorman, G. S.; Amster, I. J. Org. Mass Spectrom. 1993, 28, 437-444.

12. Gimon-Kinsel, M. E.; Kinsel, G. R.; Edmondson, R. D.; Russell, D. H. J. Am. Soc. Mass Spectrom. 1995, 6, 578.

13. Seeterlin, M. A.; Vlasak, P. R.; Beussman, D. J.; McLane, R. D.; Enke, C. G. J. Am. Soc. Mass Spectrom. 1993, 4, 751-754.

14. Gardner, B. D.; Vlasak, P. R.; Beussman, D. J.; Enke, C. G.; Watson, J. T. Proceedings of the 42nd ASMS Conference on Mass Spectrometry and Allied Topics; Chicago, IL, May 29 to June 3, 1994; p 1037.

15. Vlasak, P. R.; Beussman, D. J.; Davenport, M. R.; Enke, C. G. Rev. Sci. I., to appear.

16. Vlasak, P. R.; Beussman, D. J.; Ji, Q.; Enke, C. G. J. Am. Soc. Mass Spectrom, to appear.

17. Ross, P. L.; Johnston, M. V. I. Phys. Chem. 1993, 97, 10725-10731.

18. Van Bramer, S. E.; Ross, P. L.; Johnston, M. V. I. Am. Soc. Mass Spectrom. 1993, 4, 65-72.

19. Handbook of Chemistry and Physics, 71st ed.; Lide, D. R., Ed.; CRC Press: Boca Raton, FL, 1990; pp 9-95-9-98.

20. Yost, R. A.; Enke, C. G.; McGilvery, D. C.; Smith, D.; Morrison, J. D. Int. J. Mass Spectrom. Ion Phys. 1979, 30, 127-136. 